\title{
High expression of B23 is associated with tumorigenesis and poor prognosis in bladder urothelial carcinoma
}

\author{
HAIPING WANG, GANGJUN YUAN, BAI ZHAO, YAKUN ZHAO and YU QIU \\ Department of Urology, Second Affiliated Hospital of Harbin Medical University, Harbin, Heilongjiang 150086, P.R. China
}

Received October 23, 2015; Accepted November 10, 2016

DOI: $10.3892 / \mathrm{mmr} .2016 .6033$

\begin{abstract}
B23, a multifunctional nucleolar protein, is overexpressed in numerous cancers and is associated with tumorigenesis. However, the clinical significance and potential role of B23 in bladder urothelial carcinoma remains to be elucidated. The present study observed that the mRNA and protein expression levels of B23 were increased in bladder cancer cells and tissues. The overexpression of B23 contributed to tumorigenesis and was associated with poor prognosis in bladder cancer patients. Silencing of B23 by short hairpin RNA inhibited tumor cell growth and colony formation. In addition, knockdown of B23 suppressed the phosphorylation of extracellular signal-regulated kinase (ERK), resulting in the inactivation of the ERK signaling pathway. Therefore, the present study indicated that B23 promotes bladder cancer cell growth via activation of the ERK signaling pathway and is a novel potential biomarker for the diagnosis and prognosis of bladder cancer.
\end{abstract}

\section{Introduction}

Bladder cancer is the fourth most commonly occurring cancer worldwide and the most common genitourinary malignant cancer in China. Previous studies have demonstrated that of 5,647 newly-diagnosed bladder cancer cases in 2009, 2,223 were expected to be fatal $(1,2)$. Environmental and genetic factors are important in the development and progression of bladder cancer; however, the mechanisms underlying carcinogenesis remain to be fully elucidated. Thus, identifying potential carcinogenic genes is important to develop novel therapeutic strategies and specify novel biomarkers for the diagnosis and prognosis of bladder cancer.

B23 (also known as nucleophosmin, numatrin or NO38) is a nucleolar phosphoprotein that shuttles continuously between

Correspondence to: Dr Haiping Wang, Department of Urology, Second Affiliated Hospital of Harbin Medical University, 246 Xuefu Road, Nangang, Harbin, Heilongjiang 150086, P.R. China

E-mail: wanghaiping083@sina.com

Key words: B23, bladder urothelial carcinoma, prognosis, diagnosis, extracellular signal-regulated kinase the nucleus and the cytoplasm (3). Previous studies have indicated that B23is important in various cellular processes, including ribosome biogenesis, nucleocytoplasmic transport, centrosome duplication, apoptosis, cell proliferation, and in pathological conditions including cancer development and progression (4-6). B23 expression is increased in cancer and proliferating cells compared with healthy resting cells. The overexpression of B23 at the mRNA and protein levels contributes to tumorigenesis and is associated with poor prognosis in numerous cancers, including astrocytomas, colorectal cancer, hepatocellular carcinomas, breast cancer, ovarian carcinomas and prostate carcinomas (7-10). However, the association between the expression of B23 and survival and prognosis in bladder urothelial carcinoma remains to be elucidated.

The present study analyzed the mRNA and protein expression levels of B23 in bladder urothelial carcinoma and matched adjacent tissues. It was observed that the protein expression levels of B23 were increased in bladder urothelial carcinoma and that augmented B23 levels were associated with poor prognosis. Subsequently, the present study investigated the effect of B23 on cell growth and tumorigenesis in bladder cancer cells and observed that increased levels of B23 promoted cancer cell growth and tumorigenesis via regulation of extracellular signal-regulated kinase (ERK) phosphorylation.

\section{Materials and methods}

Clinical samples. The Second Affiliated Hospital of Harbin Medical University (Harbin, China) provided 95 well-documented surgically matching pairs of bladder urothelial carcinoma tissue samples, and the corresponding adjacent tissue samples, obtained from 2006 to 2009. The characteristics of the patients and their tumors were collected via a review of medical records and pathological reports. The patients were followed postoperatively for a mean of 81.5 months (range, 60-105 months). Informed consent was obtained from patients between 2006 and 2009. The present study was approved by the ethics committee of the Second Affiliated Hospital of Harbin Medical University. None of the patients underwent chemotherapy or radiotherapy prior to surgery, and there was no co-occurrence of other diagnosed cancers.

Sections of the dissected tumor samples were fixed in formalin and embedded in paraffin. Sections of paraffin-embedded tissue were used for immunohistochemical analysis (IHC). Further tumor samples and their corresponding 
adjacent tissue samples from resected bladders were frozen in liquid nitrogen and stored at $-80^{\circ} \mathrm{C}$ for protein and nucleic acid extraction.

IHC. Tissue samples were processed according to routine procedures. In brief, the paraffin-embedded bladder urothelial carcinoma tissue samples and the corresponding adjacent tissue samples were sectioned at $4 \mu \mathrm{m}$ and mounted on glass slides. The slides were subsequently deparaffinized, hydrated, incubated with $3 \% \mathrm{H}_{2} \mathrm{O}_{2}$ and microwaved for $20 \mathrm{~min}$ at room temperature to block endogenous peroxidase activity and expose antigens concealed by formalin fixation. Non-specific antigen-antibody reactions were inhibited using an immunohistochemistry Protein Blocker-serum and Azide Free (MB-071-0100, Rockland Immunochemicals, Inc., Pottstown, PA, USA) for $5 \mathrm{~min}$, following which the slides were washed thoroughly with PBS. The slides were subsequently incubated overnight with a rabbit polyclonal primary antibody against B23 (1:200; cat. no. 10306-1-AP; Proteintech Group, Inc., Rosemont, USA) at $4^{\circ} \mathrm{C}$. A biotinylated goat anti-rabbit secondary antibody (1:200; cat.no. ab6720; Abcam, Cambridge, UK) was applied for $20 \mathrm{~min}$ at room temperature, followed by further washing with buffer to remove any unbound antibody. A complex of avidin conjugated to horseradish peroxidase was then applied for $20 \mathrm{~min}$ at room temperature. For color development, the slides were incubated with 3,3'diaminobenzidine (Sigma-Aldrich; Merck Millipore, Darmstadt, Germany) and counterstained with hematoxylin. Staining results were evaluated using Aperio VERSA Brightfiled, Fluorescence, FISH Digital pathology scanner (Leica Microsystems, Ltd., Milton Keynes, UK) by two independent observers blinded to clinicopathological data. Regarding the cases with discordant evaluation, two pathologists performed a consensus adjudication review using a multi-headed light microscope.

Cell culture and reagents. The RT-4, BI-87,253 J, SV-HUC-1, T24 and J82 human bladder tumor cell lines were purchased from China Academia Sinica Cell Repository (Shanghai, China; www.cellbank.org.cn). T24, RT-4, and 253 J cells were cultured in RPMI 1640 (cat. no. 22400-089; Gibco; Thermo Fisher Scientific, Inc., Waltham, MA, USA) supplemented with $10 \%$ fetal calf serum (Gibco; Thermo Fisher Scientific, Inc.). J82 cells were maintained in Opti-Minimal Essential Medium ${ }^{\circledR}$ I (cat. no. 51985-042; Gibco; Thermo Fisher Scientific, Inc.) containing $10 \%$ fetal calf serum (Gibco; Thermo Fisher Scientific, Inc.). SV-HUC-1 cells were cultured in F12K medium (cat. no. N3520; Sigma-Aldrich; Merck Millipore) supplemented with $10 \%$ fetal calf serum (Gibco; Thermo Fisher Scientific, Inc.) All cells were cultured in a humidified incubator at $37^{\circ} \mathrm{C}$ and $5 \% \mathrm{CO}_{2}$. The following antibodies were used: Anti-B23 (cat. no. 10306-1-AP; Proteintech Group, Inc., Rosemont, USA), mouse anti-GAPDH (cat. no. sc-32233; Santa Cruz Biotechnology, Inc., Dallas, TX, USA), rabbit anti-ERK (cat. no. 4372S; Cell Signaling Technology, Inc., Danvers, MA, USA) and rabbit anti-phosphorylated (p)-ERK1/2 (cat. no. 4370S; Cell Signaling Technology, Inc.). U0126 (cat. no. 9903; Cell Signaling Technology, Inc.) was selected as the mitogen activated protein kinase (MAPK) /ERK inhibitor and the cells were treated with $2 \mu \mathrm{M}$ U0126 for $1,2,3$ days at $37^{\circ} \mathrm{C}$.
Lentivirus transfection. B23 short hairpin RNA(shRNA) was cloned using the PLKO.1 vector (cat. no. SHCLNG-NM002520; Sigma-Aldrich; Merck Millipore, USA); the targeting sequence was 5-GCCAAGAATGTGTTGTCCAAA-3. The empty vector PLKO.1 was used as the control. Stable knockdown T24 and J82 cells were established as previously described $(11,12)$. To generate lentivirus expressing B23, HEK 293T cells cultured on a $6 \mathrm{~cm}$ dish and were transfected with $2 \mu \mathrm{g}$ pCDH-Flag-B23 or empty vector (cat. no. CD510B-1, System Biosciences, Palo Alto, CA, USA), $1.5 \mu \mathrm{g}$ psPax 2 and $0.5 \mu \mathrm{g}$ pMD2 G. Cells were cultured with DMEM containing 10\% FBS $24 \mathrm{~h}$ after the transfection for an additional $24 \mathrm{~h}$. The culture medium containing lentiviral particles was centrifuged at $1,000 \mathrm{x} g$ for $5 \mathrm{~min}$. Viral particles collected in the supernatant were used for infection. In order to establish the stable cell line, puromycin (cat. no. P7130; Sigma-Aldrich; Merck Millipore) was used as a selection marker for the infected cells. The expression efficiency was evaluated by western blot analysis.

RNA extraction and reverse transcription-quantitative polymerase chain reaction $(R T-q P C R)$. TRIzol ${ }^{\circledR}$ (Ambion; Thermo Fisher Scientific, Inc.) was used to isolate total RNA, of which $1 \mu \mathrm{g}$ was used to synthesize cDNA with the PrimeScript $^{\mathrm{TM}}$ RT reagent kit (cat. no. DRR037A; Takara Biotechnology Co., Ltd., Dalian, China) according to the manufacturer's protocol. SYBR ${ }^{\circledR}$ Premix Ex Taq (RR430A; Takara Biotechnology Co., Ltd.) and ROX (RR430A; Takara Biotechnology Co., Ltd.) were used to perform qPCR $\left(95^{\circ} 30\right.$ sec; $60^{\circ} \mathrm{C} 60 \mathrm{sec} ; 40$ cycles), and results were analyzed with Statagene Mx3000p (Agilent Technologies, Inc., Santa Clara, CA, USA) (13). The primer sequences used for PCR were as follows: Forward, 5-CTCCATCCTGGCCTCGCTGT-3 and reverse, 5-GCTGTCACCTTCACCGTTCC-3 for actin; and forward, 5-TTCAGGGCCAGTGCATATTAG-3 and reverse, 5-TTCTGTGGAACCTTGCTACC-3 for B23.

Western blot analysis. The antibodies used for western blot analysis were diluted as followed: anti-B23 (1:1,000), mouse anti-GAPDH $(1: 5,000)$, rabbit anti-ERK $(1: 1,000)$ and rabbit anti-phosphorylated (p)-ERK1/2 (1:1,000). Total protein was extracted from bladder urothelial carcinoma and non-cancerous adjacent tissues or T24 and J82 cell lysates, for use in immunoblotting. The protocol used was as previously described (11).

Cell viability and colony formation assays. Cell viability was detected using a Cell Counting kit-8 assay (cat. no. CK04-13; Dojindo Molecular Technologies, Inc., Kumamoto, Japan). Cells were seeded in 96-well plates at a density of 5,000 cells in $100 \mu \mathrm{l}$ medium per well, $24 \mathrm{~h}$ prior to the experiment. For the colony formation assay, T24 and J82 cells were trypsinized and 1,000 viable cells were subcultured in 6-well plates in triplicate. Cells were allowed to adhere and colonize for 10 days. To visualize colonies, media was removed and cells were fixed in $96 \%$ ethanol for $10 \mathrm{~min}$ and stained with crystal violet staining solution.

Statistical analysis. Pearson's chi-square test was used to determine differences in B23 expression between bladder urothelial carcinoma tissues and the corresponding adjacent tissues, 
$\mathbf{A}$

200

$\times 400$

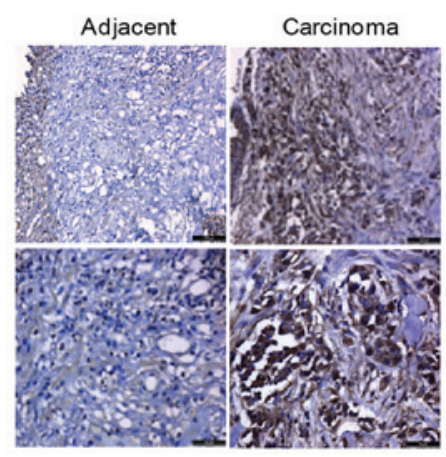

C
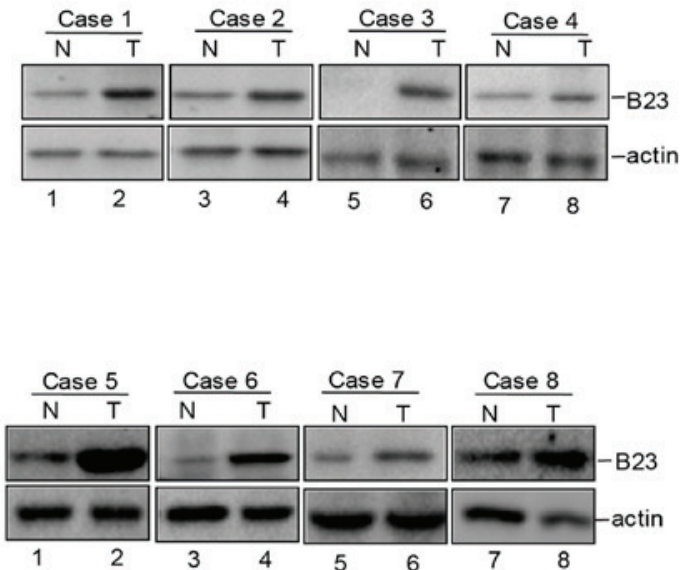

$\mathbf{E}$

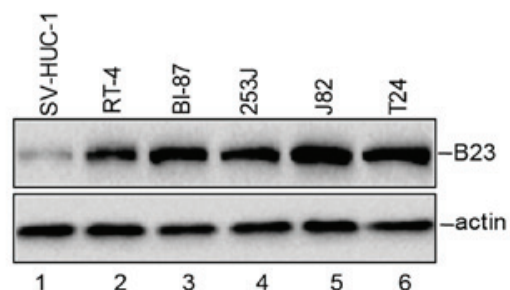

B

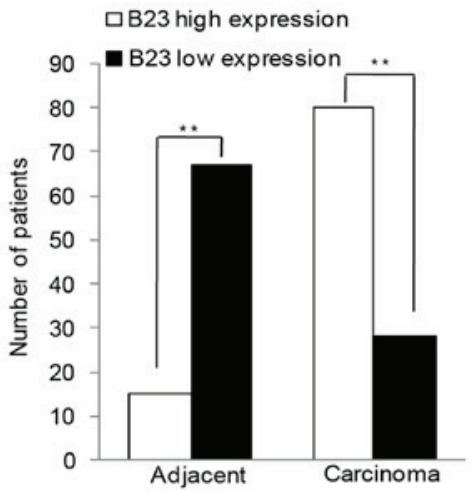

D
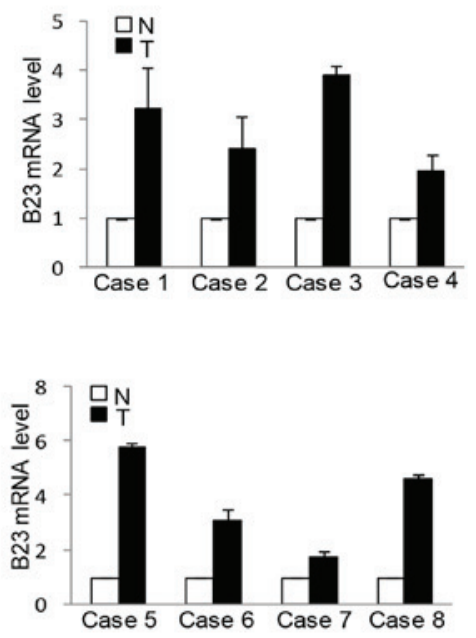

$\mathbf{F}$

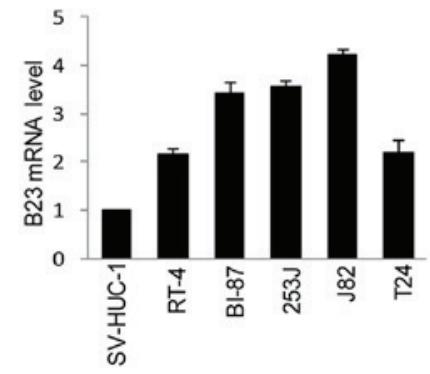

Figure 1. Expression of B23 in bladder urothelial carcinoma and adjacent non-cancerous tissues, and normal human bladder and bladder cancer cells. (A) B23 expression in carcinoma and adjacent non-cancerous tissues was detected by immunohistochemical staining. (B) The percentage of B23-positive cases in 95 tumor tissues and the matched adjacent tissues was detected by immunohistochemistry analysis. There was a statistically significant increase in B23 staining between bladder urothelial carcinoma tissues and adjacent tissues. ${ }^{* *} \mathrm{P}<0.01$. (C) Protein and (D) mRNA expression levels of B23 in normal bladder tissues and tumors from bladder urothelial carcinoma patients were detected via western blotting and RT-qPCR, respectively. Protein and mRNA expression levels of B23 were increased in carcinoma tissues compared with adjacent tissues. (E) Protein and (F) mRNA expression levels of B23 in SV-HUC-1 normal human bladder cells and RT-4, BI-87, 253 J, J82 and T24 bladder cancer cell lines were detected by western blotting and RT-qPCR, respectively. B23 was highly expressed in bladder cancer cells. RT-qPCR, reverse transcription-quantitative polymerase chain reaction; N, normal bladder tissues; T, tumor tissues.

and to determine the association between B23 expression and the clinical parameters of gender, age, tumor size, initial clinical stage, pathological grade and recurrence. Overall and disease-free survival following tumor removal was calculated by the Kaplan-Meier method, and difference in survival curves was analyzed by the log-rank test. The Cox proportional hazards model was used for multivariate analysis of prognostic factors. $\mathrm{P}<0.05$ was considered to indicate a statistically significant difference. All statistical analysis was performed using SPSS software version 16.0 (SPSS, Inc., Chicago, IL, USA).

\section{Results}

Overexpression of $B 23$ in bladder urothelial carcinoma tissues and cells. B23 expression was detected in $70.5 \%(67 / 95)$ of bladder urothelial carcinoma tissues, and in 16\% (15/95) of adjacent tissues, as assessed by IHC. The difference in B23 expression between bladder urothelial carcinoma tissues and adjacent tissues was statistically significant $(\mathrm{P}<0.01$ vs. adjacent tissues; Student's $t$-test) Fig. 1A and B). The present study detected the expression of B23 in bladder urothelial carcinoma 
A

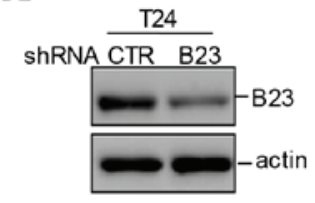

B

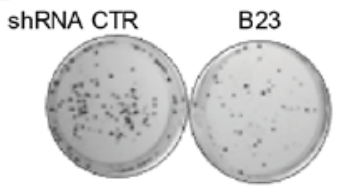

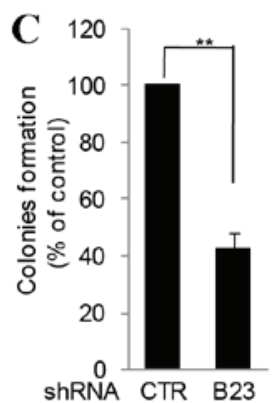

G

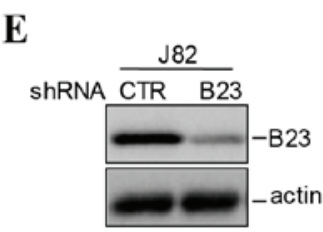

F

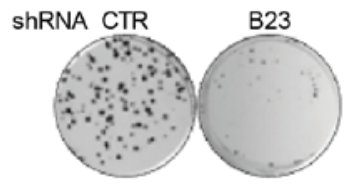

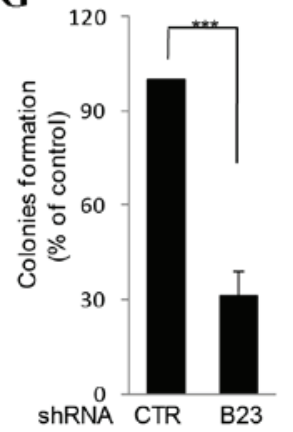

D

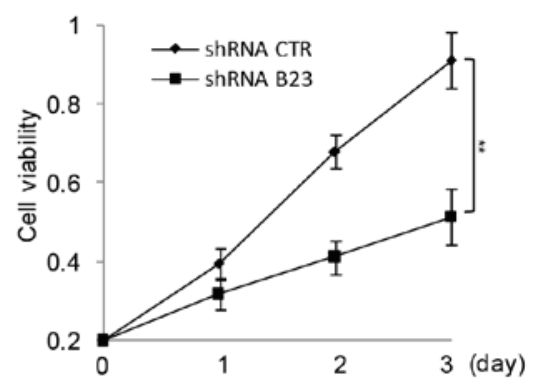

H

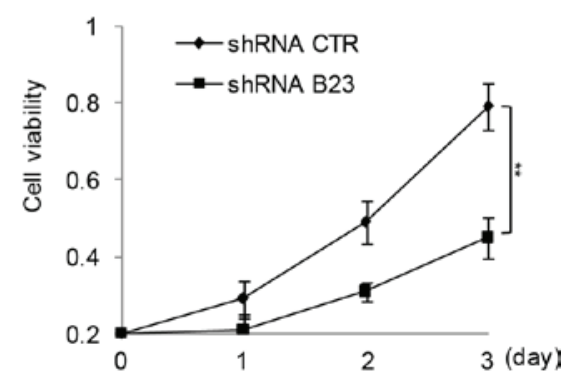

Figure 2. B23 mediates bladder cancer cell growth. (A) Western blot analysis of B23 expression following shRNA-mediated silencing of B23 expression or control shRNA treatment in the T24 human bladder urothelial carcinoma cell line. (B) A colony formation assay was used to measure the clonogenicity of T24 cells with or without knockdown of B23. (C) The number of untreated cells was set as $100 \%$. Data are expressed as the mean \pm standard deviation ( $=3$ ). ${ }^{* *} \mathrm{P}<0.01$. (D) Cell viability was measured by CCK8 assay. Data are expressed as the mean \pm standard deviation ( $\mathrm{n}=3$ ). ${ }^{* *} \mathrm{P}<0.01$ vs. shRNA CTR. (E) Western blot analysis of B23 expression following shRNA-mediated silencing of B23 expression or control shRNA treatment in the J82 human bladder urothelial carcinoma cell line. (F) A colony formation assay was used to measure the clonogenicity of J82 cells with or without knockdown of B23. (G) The number of untreated cells was set as $100 \%$. Data are expressed as the mean \pm standard deviation $(n=3)$. ${ }^{* * *} \mathrm{P}<0.001$. (H) Cell viability was measured by CCK8 assay. Data are expressed as the mean \pm standard deviation $(n=3)$. ${ }^{* *} \mathrm{P}<0.01$ vs. shRNA CTR. B23 knockdown in T24 and J82 cells decreased cell viability and colony formation compared with the control shRNA-treated cells. CTR, control; shRNA, short hairpin RNA; CCK8, Cell Counting kit-8.

tissues and their corresponding adjacent non-cancer tissues by western blot analysis and RT-qPCR, and it was observed that the protein (Fig. 1C) and mRNA (Fig. 1D) expression levels of B23 were increased in carcinoma tissues compared with adjacent tissues. The expression of B23 in bladder cancer cells and normal bladder cells was then investigated. High protein (Fig. 1E) and mRNA (Fig. 1F) expression levels of B23 were observed in the bladder cancer cells. This data suggested that the expression levels of B23 were increased in bladder urothelial carcinoma tissues and cells.

B23 promotes bladder cancer cell proliferation. Based on the data described above, the present study hypothesized that B23 may be important for bladder cancer tumorigenesis. Stable B23 knockdown cells were constructed in the T24 and J82 bladder cancer cell lines. B23 knockdown T24 and J82 cells demonstrated a dramatic decrease in cell viability and colony formation, compared with cells with the empty vector (Fig. 2).

B23 regulates bladder cancer cell growth via the ERK signaling pathway. Previous studies have reported that B23 is a positive regulator of the ERK signaling pathway and may promote cell proliferation via ERK activity in prostate cancer (14-16). Therefore, to investigate whether B23 affected bladder cancer cell growth via the ERK signaling pathway, the present study detected the protein expression levels of p-ERK, and it was observed that the phosphorylation of ERK was inhibited by B23 knockdown; however, the total protein expression levels of ERK remained unaltered (Fig. 3A). The phosphorylation of ERK was increased in B23 overexpressing bladder cancer cells (Fig. 3B). To confirm that B23 promoted bladder cancer growth via upregulation of ERK phosphorylation, T24 and J82 bladder cancer cells were treated with the pharmacological ERK inhibitor U0126. The effect of B23 on cell growth was suppressed by ERK inhibition via U0126 (Fig. 3C and D).

Association between B23 expression and overall survival rate in bladder urothelial carcinoma patients. The association between B23 expression and patient survival rate was determined using Kaplan-Meier analysis and the log-rank test. As presented in Fig. 4A, a statistical correlation was observed between B23 protein expression levels and overall survival in bladder urothelial carcinoma patients was determined by IHC. Patients with high B23 expression had a markedly lower survival rate compared with patients with low B23 expression $(\mathrm{P}<0.001)$. There was no statistical significance observed between B23 expression and other clinical parameters including age and gender (Fig. 4B and C). Subsequently, the association between B23 expression and different clinical parameters, including tumor size, initial clinical stage and tumor stage were investigated. Significant positive correlations between B23 expression and tumor size ( $\mathrm{P}=0.041$; Fig. 4D), tumor stage $(\mathrm{P}=0.030$; Fig. 4E), initial clinical stage $(\mathrm{P}=0.021)$ and recurrence $(\mathrm{P}=0.049)$ were observed. 
$\mathbf{A}$

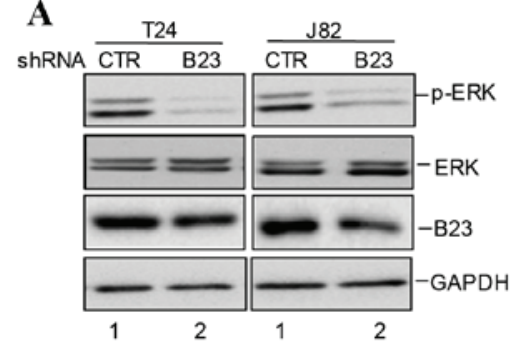

C

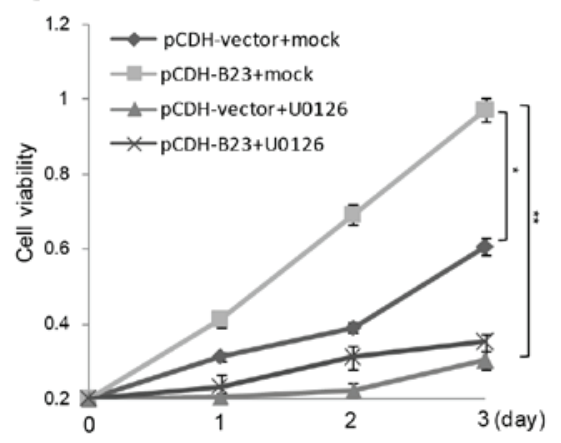

B

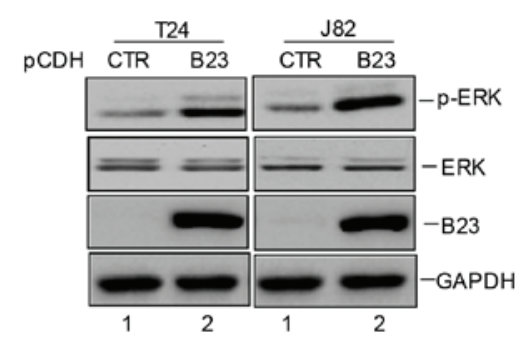

D

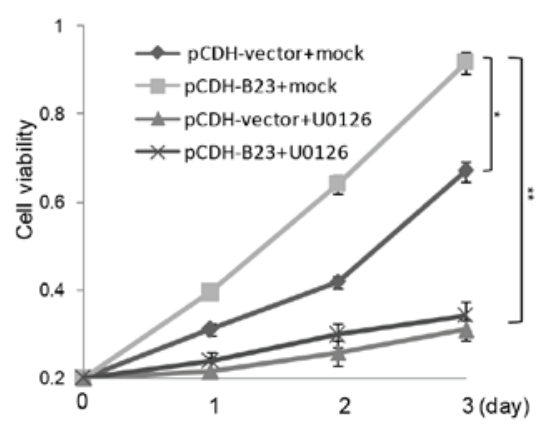

Figure 3. B23 mediates bladder cancer cell growth via the ERK signaling pathway. (A) T24 and J82 cells with or without B23 knockdown were analyzed by western blotting using the indicated antibodies. GAPDH served as the loading control. Phosphorylation of ERK was inhibited by B23 knockdown; however, the total level of ERK remained unaltered. (B) T24 and J82 cells with or without B23 overexpression were analyzed by western blotting using the indicated antibodies. GAPDH served as the loading control. Phosphorylation of ERK was increased in B23 overexpressing bladder cancer cells. (C) T24 and (D) J82 cells with or without B23 overexpression were treated with the ERK inhibitor U0126 for the indicated times. Cell viability was measured using a Cell Counting kit-8 assay. The effect of B23 on cell growth was suppressed by ERK inhibition. Data are expressed as the mean \pm standard deviation $(\mathrm{n}=3)$. "P<0.05 and ${ }^{* *} \mathrm{P}<0.01$ vs. CTR. ERK, extracellular signal-regulated kinase; p, phosphorylated; shRNA, short hairpin RNA; CTR, control.

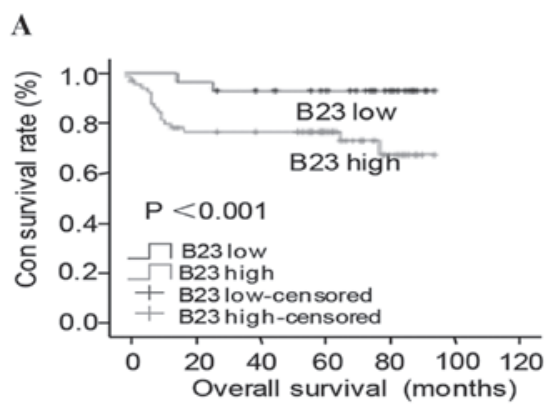

C

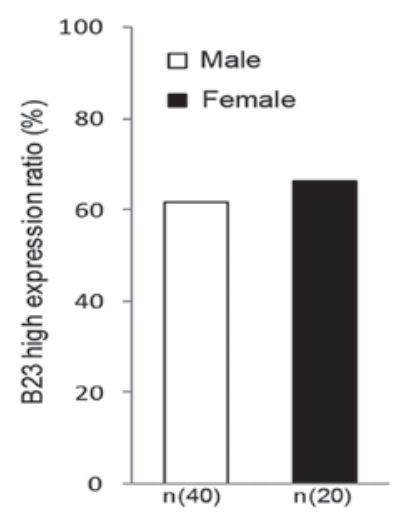

B

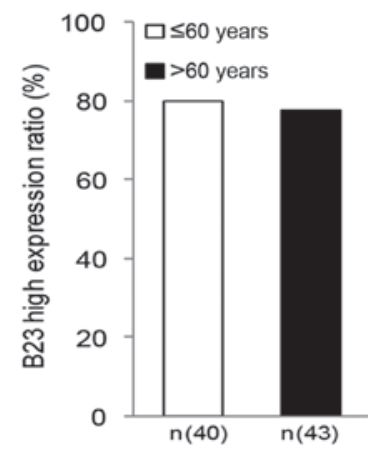

D

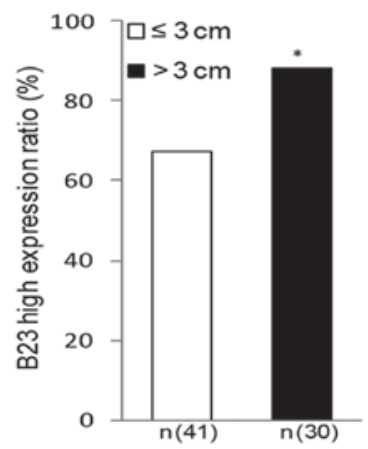

$\mathbf{E}$

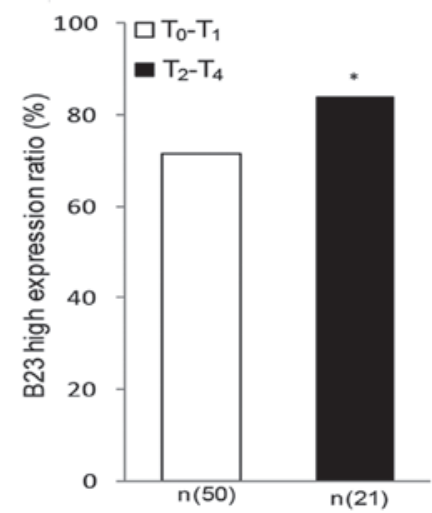

Figure 4. Clinical correlation between overall survival and B23 protein expression in 95 bladder urothelial carcinoma patients. (A) overall survival of bladder urothelial carcinoma patients with high or low B23 expression.Those with a high B23 expression demonstrated a markedly reduced survival rate compared with patients with low B23 expression. Analysis of the association of high B23 expression with (B) age and (C) gender of the patients. There were no statistically significant differences observed between B23 expression and age or gender. Analysis of the association of high B23 expression with (D) tumor size and (E) tumor stage of patients. A significant positive association between B23 expression and tumor size and stage was observed. (D) "P $<0.05$ vs. $\leq 3 \mathrm{~cm}$; (E) ${ }^{*} \mathrm{P}<0.05$ vs. T0-T1. 


\section{Discussion}

Bladder urothelial carcinoma is a common malignant cancer that results in $\sim 150,000$ fatalities per year worldwide (17). It has been suggested that environmental and genetic factors are important in the development and progression of bladder cancer; however, the underlying mechanisms of bladder carcinogenesis remain to be fully elucidated. It is important to understand these mechanisms to predict tumor behavior and identify novel therapeutic targets.

The present study reported that the mRNA and protein expression levels of B23 were increased in human bladder urothelial carcinoma tissues compared with adjacent non-cancerous tissues, and that the increased B23 expression promoted bladder cancer cell growth via the ERK signaling pathway. Following this, the association between B23 expression and prognosis in 95 bladder urothelial carcinoma patients was investigated. It was observed that augmented B23 expression was correlated with poor prognosis.

B23 is a multifunctional protein involved in a complex network of interactions. Previous studies have demonstrated the overexpression of B23 in numerous solid tumors, including gastric, prostate and liver cancer $(8-10,18)$. However, the function of B23 in bladder urothelial carcinoma remains to be elucidated. The present study, to the best of our knowledge, was the first to analyze the expression of B23 in bladder urothelial carcinoma tissues and matched adjacent tissues. It was observed that the mRNA and protein expression levels of B23 were increased in bladder urothelial carcinoma tissues compared with adjacent non-cancerous tissues. Following this, the expression of B23 in bladder cancer and normal bladder cells was investigated, and it was demonstrated that B23 was upregulated in cancer compared with normal cells. The data from the present study indicated that B23 was overexpressed in bladder urothelial carcinoma tissues and cells; however, the underlying molecular mechanism remains to be elucidated, and will be investigated in future studies.

An increase in B23 expression levels has been associated with an increase in proliferating cells, compared with normal resting cells (19). Therefore, the present study investigated whether B23 was important in bladder cancer cells. B23 knockdown T24 and J82 cells demonstrated a marked decrease in cell viability and colony formation, compared with control cells. A previous study indicated the abnormal activation of the MAPK signaling pathway was important for bladder cancer (20). To determine whether B23 was involved in the activation of the MAPK signaling pathway in bladder cancer, the present study detected the expression of phosphorylation of ERK and revealed that knockdown of B23 inhibited the activation of ERK. Following this, it was demonstrated B23 promoted bladder cancer cell growth via regulation of the MAPK pathway.

The correlation between B23 expression and prognosis in the 95 bladder urothelial carcinoma patients was subsequently investigated. A significant positive correlation was observed between B23 expression and tumor size, initial clinical stage, tumor stage and recurrence. No statistical significance was observed between B23 expression and age or gender. In addition, it was observed that patients with high B23 expression had a markedly reduced survival rate compared with patients with low B23 expression.
In conclusion, the results of the present study revealed that B23 expression levels were increased in bladder urothelial carcinoma tissues, and B23 was a reliable and independent prognostic factor for bladder urothelial carcinoma patients. In addition, it was observed that elevated B23 expression accelerated bladder cancer cell growth and tumorigenesis via the MAPK signaling pathway. Therefore, the evidence suggested that B23 may be a potential therapeutic target for the treatment of bladder urothelial carcinoma.

\section{Acknowledgements}

The present study was supported by the Health and Family Planning Commission of Heilongjiang Province (grant nos. 2007315 and 2005251).

\section{References}

1. Jemal A, Bray F, Center MM, Ferlay J, Ward E and Forman D: Global cancer statistics. CA Cancer J Clin 61: 69-90, 2011.

2. Gu F: Changing constituents of genitourinary cancer in recent 50 years in Beijing. Chin Med J (Engl) 116: 1391-1393, 2003.

3. Grisendi S, Mecucci C, Falini B and Pandolfi PP: Nucleophosmin and cancer. Nat Rev Cancer 6: 493-505, 2006.

4. Chan WY, Liu QR, Borjigin J, Busch H, Rennert OM, Tease LA and Chan PK: Characterization of the cDNA encoding human nucleophosmin and studies of its role in normal and abnormal growth. Biochemistry 28: 1033-1039, 1989.

5. Feuerstein N, Chan PK and Mond JJ: Identification of numatrin, the nuclear matrix protein associated with induction of mitogenesis, as the nucleolar protein B23. Implication for the role of the nucleolus in early transduction of mitogenic signals. J Biol Chem 263: 10608-10612, 1988.

6. Hingorani K, Szebeni A and Olson MO: Mapping the functional domains of nucleolar protein B23. J Biol Chem 275: 24451-24457, 2000.

7. Gimenez M, Souza VC, Izumi C, Barbieri MR, Chammas R, Oba-Shinjo SM, Uno M, Marie SK and Rosa JC: Proteomic analysis of low- to high-grade astrocytomas reveals an alteration of the expression level of raf kinase inhibitor protein and nucleophosmin. Proteomics 10: 2812-2821, 2010.

8. Nozawa Y, Van Belzen N, Van der Made AC, Dinjens WN and Bosman FT: Expression of nucleophosmin/B23 in normal and neoplastic colorectal mucosa. J Pathol 178: 48-52, 1996.

9. Skaar TC, Prasad SC, Sharareh S, Lippman ME, Brünner N and Clarke R: Two-dimensional gel electrophoresis analyses identify nucleophosmin as an estrogen regulated protein associated with acquired estrogen-independence in human breast cancer cells. J Steroid Biochem Mol Biol 67: 391-402, 1998.

10. Yun JP, Miao J, Chen GG, Tian QH, Zhang CQ, Xiang J, Fu J and Lai PB: Increased expression of nucleophosmin/B23 in hepatocellular carcinoma and correlation with clinicopathological parameters. Br J Cancer 96: 477-484, 2007.

11. Han C, Gu H, Wang J, Lu W, Mei Y and Wu M: Regulation of L-threonine dehydrogenase in somatic cell reprogramming. Stem Cells 31: 953-965, 2013.

12. Han C, Jin L, Mei Y and Wu M: Endoplasmic reticulum stress inhibits cell cycle progression via induction of p27 in melanoma cells. Cell Signal 25: 144-149, 2013.

13. Livak KJ and Schmittgen TD: Analysis of relative gene expression data using real-time quantitative PCR and the 2(-Delata C(T)) Method. Methods 11: 402-408, 2001.

14. Loubeau G, Boudra R, Maquaire S, Lours-Calet C, Beaudoin C, Verrelle $\mathrm{P}$ and Morel L: NPM1 silencing reduces tumour growth and MAPK signalling in prostate cancer cells. PLoS One 9: e96293, 2014.

15. Yang X, Du T, Wang X, Zhang Y, Hu W, Du X, Miao L and Han C: IDH1, a CHOP and C/EBP $\beta$-responsive gene under ER stress, sensitizes human melanoma cells to hypoxia-induced apoptosis. Cancer Lett 365: 201-210, 2015.

16. Zhang D, Zhu L, Li C, Mu J, Fu Y, Zhu Q, Zhou Z, Liu P and Han C: Sialyltransferase7A, a Klf4-responsive gene, promotes cardiomyocyte apoptosis during myocardial infarction. Basic Res Cardiol 110: 28, 2015. 
17. Mitra AP and Cote RJ: Molecular pathogenesis and diagnostics of bladder cancer. Annu Rev Pathol 4: 251-285, 2009.

18. Mina N, Soubani AO, Cote ML, Suwan T, Wenzlaff AS, Jhajhria S, Samarah H and Schwartz AG: The relationship between chronic obstructive pulmonary disease and lung cancer in African American patients. Clin Lung Cancer 13: 149-156, 2012.
19. Chen J, Sun J, Yang L, Yan Y, Shi W, Shi J, Huang Q, Chen J and Lan Q: Upregulation of of B23 promotes tumor cell proliferation and predicts poor prognosis in glioma. Biochemn Biophys Res Commum 10: 124-130, 2015.

20. Koul HK, Pal M and Koul S: Role of p38 MAP kinase signal transduction in solid tumors 9: 2013, 342-359. 